\title{
Inhibition of Defocus-Induced Myopia in Chickens
}

\author{
Jill Woods, ${ }^{1}$ Sarah E. Guthrie, ${ }^{1}$ Nancy Keir, ${ }^{1}$ Sally Dillehay, ${ }^{2}$ Mark Tyson, ${ }^{2}$ Richard Griffin, ${ }^{2}$ \\ Vivian Choh, ${ }^{3}$ Desmond Fonn, ${ }^{1}$ Lyndon Jones, ${ }^{1}$ and Elizabeth Irving ${ }^{3}$ \\ ${ }^{1}$ Centre for Contact Lens Research, School of Optometry \& Vision Science, University of Waterloo, Ontario, Canada \\ ${ }^{2}$ Visioneering Technologies, Inc., Alpharetta, Georgia \\ ${ }^{3}$ School of Optometry \& Vision Science, University of Waterloo, Ontario, Canada
}

Correspondence: Jill Woods, Centre for Contact Lens Research, School of Optometry \& Vision Science, University of Waterloo, 200 University Avenue West, Waterloo, ON, Canada N2L 3G1; jwoods@uwaterloo.ca.

Submitted: August 9, 2012

Accepted: February 25, 2013

Citation: Woods J, Guthrie SE, Keir N, et al. Inhibition of defocus-induced myopia in chickens. Invest Ophthalmol Vis Sci. 2013;54:2662-2668. DOI:10.1167/iovs.12-10742
Purpose. To determine the effect of wearing a lens with a unique peripheral optical design on the development and progression of defocus-induced myopia in newly hatched chickens.

Methods. Eighty-five newly hatched chickens underwent bilateral retinoscopy and A-scan ultrasound to determine their refractive error and axial length. They were randomly divided into Control and two Test groups, in which each chicken was fitted with a goggle-lens over the right eye, with the left eye remaining untreated. The Control group wore a lens of power -10.00 diopters (D) of standard spherical optical design. The two Test lenses both had a central optical power $-10.00 \mathrm{D}$, but used different peripheral myopia progression control (MPC) designs. For all groups, retinoscopy was repeated on days 3, 7, 10, and 14; ultrasound was repeated on day 14 .

Results. On day 0 there was no statistical difference in refractive error (mean $+6.92 \mathrm{D}$ ) or axial length (mean $8.06 \mathrm{~mm}$ ) between Test and Control groups or treated and untreated eyes (all $P>0.05$ ). At day 14,37 (43.5\%) of 85 chickens had not experienced goggle detachment and were included in the final analyses. In this cohort there was a significant refractive difference between the treated eyes of the Control group $(n=17)$ and those of Test $1(n=$ $14)$ and Test $2(n=6)$ groups (both $P<0.01)$ : Control $-4.65 \pm 2.11 \mathrm{D}$, Test $1+4.57 \pm 3.11$ $\mathrm{D}$, Test $2+1.08 \pm 1.24 \mathrm{D}$ (mean \pm SEM). There was also a significant axial length difference (both $P<0.01$ ): Control $10.55 \pm 0.36 \mathrm{~mm}$, Test $19.99 \pm 0.14 \mathrm{~mm}$, Test $210.17 \pm 0.18 \mathrm{~mm}$.

Conclusions. Use of these unique MPC lens designs over 14 days caused a significant reduction in the development of defocus-induced myopia in chickens; the degree of reduction appeared to be design specific.

Keywords: myopia control, emmetropization, refractive error, eye growth, multifocal lens
$\mathrm{U}$ ncorrected refractive error has been identified as a leading cause of preventable blindness in the world today. ${ }^{1}$ Although optically correctable with lenses or refractive surgery, myopia, or near-sightedness, is associated with an increase in axial length of the eye. It can be progressive in nature and high levels of myopia are associated with sight-threatening conditions. $^{2-4}$ The prevalence of myopia is reported to be increasing worldwide ${ }^{-7}$ and this has led to renewed interest in understanding and controlling the underlying mechanism of myopia progression. To date, various strategies to prevent or slow the progression of myopia in humans, such as bifocals, undercorrection, and cycloplegia, have generally either been unsuccessful in the long term, or given rise to unacceptable side effects. ${ }^{8-13}$

A great deal of information has been learned about refractive error development using various animal models. ${ }^{14-26}$ It has been established that the visual stimulus environment alters refractive development in primates, tree shrews, and chickens. ${ }^{14-20}$ Chickens are a useful animal model in which to study myopia progression, ${ }^{21}$ as they are precocial animals with a short developmental period of just a few weeks and they are relatively easy to handle to measure their refractive state with retinoscopy. Previous work with chickens, in particular, has demonstrated that defocusing the eye of a newly hatched chicken with a negative power causes the eye to compensate for the defocusing lens. ${ }^{16,17}$ This process of emmetropization, by which an eye becomes altered to attain a final refraction through the lens that approaches zero, has been shown to occur in a fairly predictable manner with a wide range of defocus stimuli, including both positively and negatively powered stimuli. ${ }^{17-20}$ The refractive change in response to negatively-powered defocusing lenses is primarily due to a change in axial length. ${ }^{18-20,22}$ Subsequent removal of the negatively powered defocus lens from the eye eventually results in the eye's refractive error returning to match that of the contralateral untreated eye. This response is initially due to choroidal thickening followed by scleral changes, which are the same processes that occur in response to positively powered defocus lenses. ${ }^{19,23,24}$

The results of recent animal experiments have led researchers to suggest that peripheral retinal focus, relative to the focus at the posterior pole, may play a role in the development of refractive error. Plotting the image shell of a negative-power corrective lens illustrates the presence of hyperopic defocus at the peripheral retina, relative to the correct focus at the central retina. This led to the theory that the relative hyperopic defocus at the peripheral retina may actively encourage the peripheral retina to shift posteriorly to create better peripheral focus, which in turn effectively increases central axial length and therefore central myopia. ${ }^{25,26}$ 
This theory has led to the development of corrective lenses with a peripheral design aimed at eliminating the relative hyperopic defocus at the peripheral retina while maintaining correct focus at the fovea. Tabernero et al. ${ }^{27}$ used a novel autorefractor and established that a negatively powered correcting lens with a positive, radial power gradient produced relative myopic defocus in the peripheral retina, instead of the relative hyperopic defocus produced by a traditional negative lens design.

The purpose of this study was to investigate the performance of new lens designs that were designed specifically for the purpose of myopia progression control (MPC) by modifying the focus in the peripheral retina. To test the hypothesis that this unique lens design would have an inhibitory effect on the development and/or progression of central myopia, defocus-induced myopia in chickens was used as a predictable "controlled" response against which the effect of the new MPC lens designs could be gauged.

\section{METHODS}

The use and treatment of the chickens in this study complied with the ARVO Statement for the Use of Animals in Ophthalmic and Vision Research, as well as the Canadian Council on Animal Care. Approval for this study was provided by the University of Waterloo's Animal Care Committee.

Eighty-five newly hatched broiler chickens (Ross-Ross strain) underwent central refractive assessment of both eyes using streak retinoscopy to within \pm 0.50 diopters (D). High frequency A-scan ultrasonography was used to measure the ocular axial lengths (anterior cornea to retina) on a subset of these chickens. Ultrasound was conducted under general anesthesia using $1.5 \%$ isoflurane in oxygen.

The chickens were then divided randomly into a Control group ( $n=37)$, a Test 1 group $(n=23)$, and a Test 2 group $(n=$ 25). Every chicken had a lens fixed unilaterally over its right eye at a vertex distance of $5 \mathrm{~mm}$ using rings of Velcro, which were glued to the chicken feathers using cyano-acrylic glue. ${ }^{22}$ For all chickens, the contralateral eye remained untreated.

To measure central refractive error, streak retinoscopy was conducted on the day of hatching (day 0 ) and repeated on days $3,7,10$, and 14 by an investigator who was masked from the Control and Test groups' assignment. Lenses were removed briefly to allow this measurement. On day 14 following retinoscopy, the axial length of each eye was measured by a masked investigator for a second time using high-frequency Ascan ultrasonography. Immediately following euthanasia, the chicken heads were flash frozen in ethanol and dry ice, then mounted in a freezing microtome and sliced into sections. Each section that provided a cross-section of the ocular globes was photographed alongside a millimeter rule.

Throughout the study period of 14 days, the chickens were maintained in stainless steel brooders with an initial temperature of $32^{\circ} \mathrm{C}$, provided with food and water ad libitum, and raised on a 14-hour light/10-hour dark cycle. The chickens were monitored every 4 hours through the day, as well as early morning and before the lights went out. Lenses were checked routinely for centration and cleanliness and removed briefly for repositioning and cleaning if deemed necessary, using new rings of Velcro when required. In cases in which the lenses may have been detached for more than 4 hours, or when they were not able to be reattached due to insufficient feathers around the eye, the chicken was euthanized and the data set was removed from the final analysis.

Comparisons were made between days and between lens designs. Repeated measures ANOVA, paired and unpaired $t$ tests with Bonferroni corrected post hoc testing with statistical significance set at $P<0.05$, were used for data analysis. Refractive error and axial length data are reported as means and SEMs. The refractive error and axial length data, as well as the difference between the treated and untreated eyes for each group (untreated value subtracted from treated value), are shown. The difference data has previously been reported this way to avoid skewing of the absolute means due to small eye artifacts. ${ }^{28}$ Thus, the mean refractive error interocular difference between eyes (MDiff ref $_{\text {) }}$ and mean axial length interocular difference between eyes (MDiff ${ }_{\text {axial }}$ ) are also reported.

\section{Lens Details}

The Control group wore a standard design $-10.00-\mathrm{D}$ spherical power lens. The Test groups wore two separate, unique MPC lens designs, which both had a central power verified to be within $\pm 0.50 \mathrm{D}$ of $-10.00 \mathrm{D}$ (in accordance with the American National Standards Institute) using a 5-mm aperture on a standard lensometer (Nikon PL 2 \#31216; Nikon Instruments Inc., Melville, NY). Both Test designs exhibited a continuous gradient of relatively positive power change extending into the periphery (US patents 6474814 and 7178918 , held by Visioneering Technologies, Inc., Alpharetta, GA). The lens designs were scaled for a back vertex distance of $5 \mathrm{~mm}$ and a $2.5-\mathrm{mm}$ chicken pupil. The Test 1 lens design incorporated a +2.75-D power rise at the pupil edge. The Test 2 lens design incorporated a slower progression in power through the periphery than the Test 1 lens. The Test 2 lens design resulted in $a+1.32-\mathrm{D}$ power rise at the pupil edge. The Control and Test lenses were identical in every aspect except for the inclusion of the peripheral MPC lens design in the Test lenses. The physical parameters of all lenses were overall diameter $20 \mathrm{~mm}$, optic zone diameter $15 \mathrm{~mm}$, and base curve radius of $10 \mathrm{~mm}$. All lenses were lathe manufactured from polymethylmethacrylate buttons obtained from Lagado Corporation (Englewood, $\mathrm{CO})$.

\section{Results}

\section{Refractive Error}

The mean refractive errors $( \pm \mathrm{SE}$ ) for the total number of chickens available at each measurement stage are shown in Table 1. The mean refractive error of the newly hatched chickens on day 0 was $6.92 \mathrm{D}$, which is similar to that reported previously. $^{29}$ There was no statistical difference at day 0 for refractive error of the Control group chickens compared with either Test group, or between treated and untreated eyes in each group (all $P>0.05$ ).

Throughout the study period, some lenses became detached from the chickens' feathers and, if the period of lens loss could not be confirmed as less than 4 hours, this chicken no longer continued in the study. This resulted in reducing sample sizes with age, as shown in Table 1.

After day 0, statistical analysis between groups was conducted on the data acquired from only those chickens who completed day 14: Control group $n=17$, Test 1 group $n=$ 14 , Test 2 group $n=6$. The mean refractive error data on each measurement day, from only the set of chickens that maintained goggles for 14 days, are presented in Figure 1.

Over the study period, the untreated eyes of all groups showed a mean reduction in hyperopia of $3.73 \mathrm{D}$, as shown by the dashed lines in Figure 1, which is consistent with expected normal development. ${ }^{29}$ There was no statistical difference $(P>0.05)$ between the untreated eyes of the three groups on any of the measurement days. Analyzing the data from only those chickens that completed the day 14 
TABLE 1. Mean Refractive Error \pm SE (D) of the Treated and Untreated Eyes of the Chickens in the Control Group, the Test 1 Group, and the Test 2 Group

\begin{tabular}{|c|c|c|c|c|c|}
\hline Group & Day 0 & Day 3 & Day 7 & Day 10 & Day 14 \\
\hline \multicolumn{6}{|l|}{ Control } \\
\hline Sample size & $n=37$ & $n=37$ & $n=29$ & $n=23$ & $n=17$ \\
\hline Treated eye & $7.15 \pm 1.65$ & $0.78 \pm 2.75$ & $-3.03 \pm 2.20$ & $-4.28 \pm 1.72$ & $-4.65 \pm 2.11$ \\
\hline Untreated eye & $6.93 \pm 1.73$ & $5.58 \pm 0.85$ & $4.67 \pm 1.10$ & $3.85 \pm 0.65$ & $2.85 \pm 0.61$ \\
\hline \multicolumn{6}{|l|}{ Test 1} \\
\hline Sample size & $n=23$ & $n=22$ & $n=19$ & $n=17$ & $n=14$ \\
\hline Treated eye & $6.78 \pm 1.44$ & $6.84 \pm 2.03$ & $6.21 \pm 2.46$ & $6.26 \pm 2.44$ & $4.57 \pm 3.11$ \\
\hline Untreated eye & $6.91 \pm 1.35$ & $5.89 \pm 0.96$ & $4.42 \pm 1.19$ & $3.26 \pm 0.73$ & $2.64 \pm 0.84$ \\
\hline \multicolumn{6}{|l|}{ Test 2} \\
\hline Sample size & $n=25$ & $n=25$ & $n=15$ & $n=13$ & $n=6$ \\
\hline Treated eye & $6.82 \pm 1.98$ & $4.36 \pm 1.45$ & $2.67 \pm 1.21$ & $2.31 \pm 0.83$ & $1.08 \pm 1.24$ \\
\hline Untreated eye & $6.78 \pm 2.07$ & $5.40 \pm 1.00$ & $4.47 \pm 0.77$ & $3.31 \pm 0.43$ & $2.83 \pm 0.41$ \\
\hline
\end{tabular}

Data from all chickens available on each measurement day are presented, although the group sizes reduced over the study period as lenses became detached from the chickens.

measurements, the treated eyes of the Control group exhibited a significant myopic progression of $11.44 \mathrm{D}$, resulting in a day 14 mean refractive error of $-4.65 \mathrm{D}$. The eyes treated with Test 1 lenses underwent a hyperopic reduction of $2.43 \mathrm{D}$, resulting in a day 14 mean refractive error of $+4.57 \mathrm{D}$ and those eyes wearing Test 2 lenses underwent a hyperopic reduction of $5.25 \mathrm{D}$, resulting in a day 14 mean refractive error of $+1.08 \mathrm{D}$. There was a statistically significant difference between the day 14 refractive error of the Control group compared with the Test 1 group $(P<0.01)$ and the Test 2 group $(P<0.01)$.

Table 2 shows the MDiff $_{\text {ref }}$ data (refractive error of the treated eye minus that of the untreated eye) for the chickens that wore a lens throughout the entire study period. There was no difference in MDiff $_{\text {ref }}$ between the Control and Test groups at day 0 (all $P>0.99$ ). Statistical differences between the Control group and both Test groups were evident from day 3 onward; only the Control group showed a statistically different MDiff $_{\text {ref }}$ at day 14 compared with day 0 . At day 14 there was a statistically significant difference between the MDiff $_{\text {ref }}$ of the Control group and the MDiff $_{\text {ref }}$ of the Test 1 group $(P<0.01)$ and the Test 2 group $(P<0.01)$. The MDiff $r$ ref of the Test 1

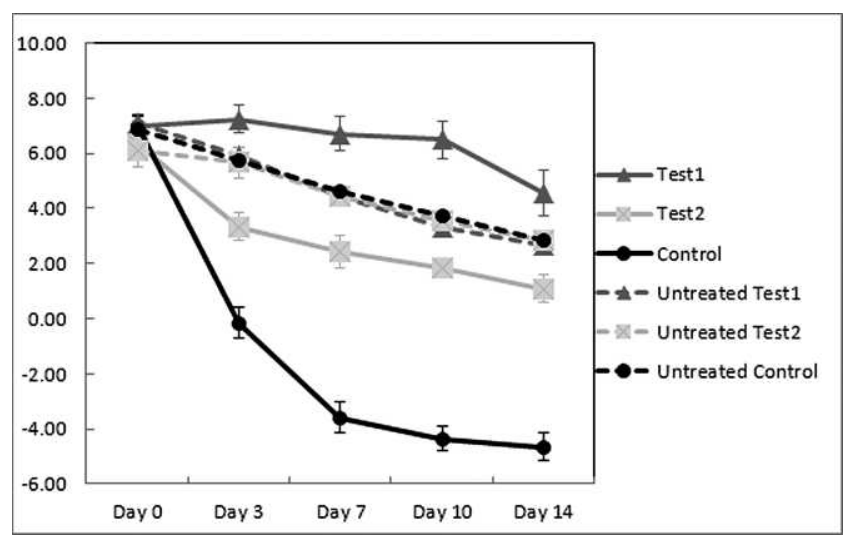

Figure 1. Mean refractive error \pm SE (D) of the treated and untreated eyes of the chickens that completed the study period from the Control group ( $n=17)$, the Test 1 group $(n=14)$, and the Test 2 group $(n=6)$. Dashed lines on graph indicate data from untreated, nonlens wearing eyes. group was not statistically significantly different from that of the Test 2 group $(P=0.90)$.

\section{Axial Length: Ultrasound}

It was not always possible to attain a clear ultrasound scan from every chicken, as the eye sometimes rotated to an extreme position, which made it impossible to identify the peaks of the scan. Therefore, the sample sizes for ultrasound are lower than those of retinoscopy for the Control and Test 1 groups. Figure 2 shows the mean axial length data for those chickens that had ultrasound measurements on both day 0 and day 14. The day 0 axial length data were not statistically different between groups, nor between the treated and untreated eyes within the groups, mean $8.06 \mathrm{~mm}$ (all $P>$ 0.05). There was also no difference in axial length between the untreated eyes of all groups at day $14(P>0.05)$ and all underwent a small increase in axial length consistent with expected normal development. ${ }^{29}$ The treated eyes of the Control group exhibited a statistically significant increase in axial length of $0.64 \mathrm{~mm}(P<0.01)$. The axial length of the treated eyes of the Test 1 and Test 2 groups also increased; however, the change was not statistically significant (both $P>$ 0.05). The day 14 axial length of the Control group was statistically significantly longer than the Test 1 and the Test 2 groups (both $P<0.01$ ).

Table 3 shows the MDiff ${ }_{\text {axial }}$ data (axial length of the treated eye minus that of the untreated eye) for the chickens measured at both day 0 and day 14 . There was no difference in MDiffaxial between the Control and Test groups at day 0 (all $P>0.99$ ). The results show that the MDiff $_{\text {axial }}$ of the Control group changed significantly over time $(P<0.01)$, whereas the MDiff $_{\text {axial }}$ of the two Test groups did not (both $P>0.05$ ). At day 14, the MDiffaxial of the Control group was statistically significantly greater than the Test 1 and Test 2 groups (both $P$ $<0.01)$. The MDiff ${ }_{\text {axial }}$ of the Test 1 group was not statistically significantly different from that of the Test 2 group $(P>0.99)$.

\section{Axial Length: Frozen Section}

Ocular sectioning of flash-frozen chicken heads was possible only in a subset of chickens due to time restrictions. However, the data serve to support the axial length data measured by ultrasound. The axial lengths measured from these chickens are presented in Figure 2. In the Control group, the treated eyes were longer than the untreated eyes $(P=0.02)$. In the Test 
Table 2. Mean Interocular Difference in Refractive Error, MDiff ref, $_{\text {. }} \pm$ SE (D), Represents the Mean of the Difference Between the Treated Eyes and the Untreated Eyes (Treated Value Minus Untreated Value)

\begin{tabular}{lcccccc}
\hline MDiff $_{\text {ref }}$ of Group & No. of Chickens & Day $\mathbf{0}$ & Day 3 & Day 7 & Day 10 & Day 14 \\
\hline Control & 17 & $0.09 \pm 0.18$ & $-5.84 \pm 0.50^{*}$ & $-8.44 \pm 0.49^{*}$ & $-8.06 \pm 0.47^{*}$ & $-7.47 \pm 0.49^{*}$ \\
Test 1 & 14 & $-0.11 \pm 0.18$ & $1.36 \pm 0.48$ & $2.29 \pm 0.65^{*}$ & $3.18 \pm 0.59^{*}$ & $1.93 \pm 0.76$ \\
Control vs. Test 1 & & $P>0.99$ & $P<0.01$ & $P<0.01$ & $P<0.01$ & $P<0.01$ \\
Test 2 & 6 & $0.25 \pm 0.42$ & $-2.33 \pm 1.21$ & $-2.00 \pm 1.30$ & $-1.75 \pm 0.52$ & $-1.75 \pm 1.29$ \\
Control vs. Test 2 & & $P>0.99$ & $P<0.01$ & $P<0.01$ & $P<0.01$ & $P<0.01$ \\
\hline
\end{tabular}

${ }^{*}$ Indicates MDiff ${ }_{\text {ref }}$ was statistically significantly different from day 0 , within group $(P<0.05)$.

1 group, there was no difference in axial length between the treated and untreated eyes $(P>0.05)$. The eyes treated with the Test 1 lens had statistically shorter axial lengths than those eyes treated with the Control lens $(P=0.02)$, whereas there was no difference between the untreated eyes of these groups $(P>0.05)$.

\section{Discussion}

The MDiff ${ }_{\text {ref }}$ data from this study illustrate that the treated eyes of the Control group responded differently from those of the Test groups. This difference was evident as early as day 3 and the difference remained statistically significant, despite the decrease in sample size over the 2 weeks. The sample size was reduced as a result of removal of chickens from all groups due to the dislodgement of their goggle and the likelihood of an immediate choroidal thickening response. The results would have been more compelling if the sample sizes had remained higher, but the overall effect is very strong despite the lower numbers on day 14. Lens dislodgement appeared to be due to molting, as the chickens lost their downy feathers from hatching to day 14, and there is no evidence that this was biased toward one batch of birds over another. As anticipated, the Control treated eyes became far more myopic compared with their contralateral untreated eye. The rapid change in the Control treated eyes during the first week, along with a slower rate of response after that period, has been reported previously. $^{30}$ The Test 1 and Test 2 treated eyes, however, showed a more moderate variation from their contralateral untreated eyes.

The measurements of axial length provide an indication of the mechanism underlying the changes in refractive state of the

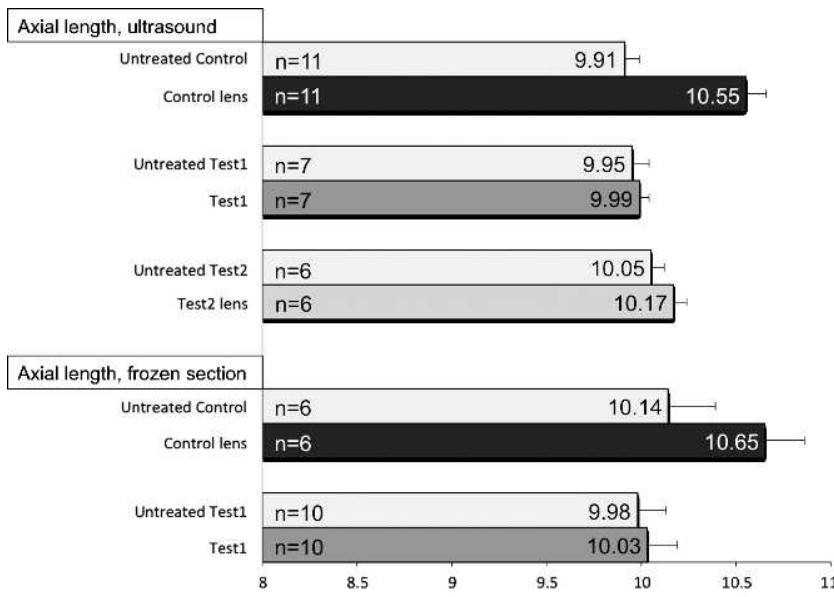

Figure 2. Mean axial length $\pm \mathrm{SE}(\mathrm{mm})$ of the treated and untreated eyes of the chickens in the Control group, the Test 1 group, and the Test 2 group at Day 14, measured by ultrasound and frozen section. chicken eyes. The axial length data measured from the frozen sections of the globes of a subgroup of the chickens corroborates the data measured using ultrasound. These results indicate that the Control lens-wearing eyes became significantly longer both compared with the untreated eyes and treated eyes of both Test groups. This was not surprising, as previous work has demonstrated that defocus-induced myopia demonstrated by the Control lens-wearing eyes would be associated with a significant increase in axial length. ${ }^{18-20,22}$ The lack of significant axial length increase seen with the Test 1 and Test 2 defocus lenses indicates that these lens designs reduced defocus-induced myopia progression through the inhibition of axial elongation.

A lens design with a modified peripheral focus relative to central focus is a logical step, as various studies in animal models have pointed to peripheral retinal focus being an important factor in refractive error development. ${ }^{24,26,31-37}$ Smith et al., ${ }^{26}$ in particular, argued for a role of the peripheral retina in central refractive control when they showed that, in primates with and without functioning central retina, negative defocus lenses applied in a peripheral annulus still led to myopia development. Stone et al. ${ }^{31}$ established that different sizes of central or peripheral apertures in opaque goggles led to varying levels of myopia and increases in axial length in chickens. In contrast, Schippert and Schaeffel, ${ }^{34}$ using powered lenses with central holes of $4 \mathrm{~mm}$ or larger, found no lensinduced ametropia . Smith et al. ${ }^{26}$ argued that this lack of effect could be explained by the vertex distance, which was such that only the extreme peripheral retina received defocus.

Liu and Wildsoet ${ }^{33,35}$ investigated chickens' responses to lenses with a different central power to peripheral power. Their results indicated some variation in the myopia progression and axial length, dependent on aperture size and the relationship of the central to the peripheral zone power. Central apertures less than $6 \mathrm{~mm}$ reduced defocus-induced myopia when the peripheral zone was more positively powered than the central zone. The level of myopia inhibition that they were able to show was less than that found in this study, but nevertheless supports the theory of relatively positive peripheral lens power playing a role in myopia control

TABLE 3. Mean Interocular Difference in Axial Length (MDiff $\left.{ }_{\text {axial }}\right) \pm \mathrm{SE}$ $(\mathrm{mm})$ of the Control Group and the Test Group Chickens, Measured by Ultrasound (Treated Value Minus Untreated Value)

\begin{tabular}{|c|c|c|c|}
\hline MDiff $_{\text {axial }}$ of Group & $\begin{array}{c}\text { No. of } \\
\text { Chickens }\end{array}$ & Day 0 & Day 14 \\
\hline Control & 11 & $-0.07 \pm 0.05$ & $0.64 \pm 0.07^{*}$ \\
\hline Test 1 & 7 & $-0.01 \pm 0.10$ & $0.04 \pm 0.06$ \\
\hline Control vs. Test 1 & & $P>0.99$ & $P<0.01$ \\
\hline Test 2 & 6 & $-0.10 \pm 0.04$ & $0.11 \pm 0.06$ \\
\hline Control vs. Test 2 & & $P>0.99$ & $P<0.01$ \\
\hline
\end{tabular}

${ }^{*}$ MDiff $_{\text {ref }}$ statistically significantly different from day 0 , within group $(P<0.05)$ 
in chickens. More recently, Tepelus et al. ${ }^{36}$ investigated the effect of two designs of plano-center radial gradient lenses on eye shape and refraction in chickens. The design with the narrower central zone and shallower peripheral power change caused hyperopic shifts both centrally and peripherally, whereas the design with the wider central zone had no effect on central refraction.

The size of the effect demonstrated in this experiment, that is, the relatively small refractive change in response to the $-10.00-\mathrm{D}$ Test 1 and Test 2 lens designs compared with the large change induced by the $-10.00-\mathrm{D}$ Control lens, is far greater than in any other published reports for chickens. The larger effect could be due to one or more of the following factors: different strains of chickens, the fact that we started treatment on the day of hatching rather than using older chickens, or specific aspects of the test lens designs.

One of the unique design aspects is that the center to periphery power profile is a gradient, which is different from the majority of lenses used previously in animal work, which have had well-defined zones or apertures. The Test 2 lens design provided a slower progression in plus power through the periphery than the Test 1 lens design. This was the only difference between the two test designs and therefore it appears that this aspect of the lens design must in some way have influenced the axial length and refractive error outcome, relative to the untreated eyes. The exact nature of this influence is unknown but it may be that the power progression inhibits (Test 1 lenses) or reduces (Test 2 lenses) emmetropization, either because it is just not necessary or because the signal is no longer detectable. Alternatively, the rate of change of the power profile may differentially affect integration across the retina between the two lens designs. Peripheral refractions were not measured in this study but, if they had been, they may have provided more insight into the mechanism involved in the inhibition of the defocus-induced myopia.

The concept of the effect being due to absolute myopic defocus in the peripheral retina may not apply here. The central retina was defocused by $-10.00 \mathrm{D}$ and therefore the peripheral image shell of the test lenses, although less hyperopic than the center, may not become anterior enough to provide peripheral myopic defocus. This will depend on factors such as eye shape relative to the image shell and what, if any, accommodation is exerted to overcome the $-10.00-\mathrm{D}$ central power of the lens. It is unlikely that the chicken will accommodate through the $-10.00-\mathrm{D}$ lens, preferring instead to view through the untreated eye. ${ }^{22,38,39}$ However, it is true that whether the eye is in the rested state or the accommodated state, compared with the central retina, the peripheral retina would have relative myopic defocus. In other words, the apparent control of the defocus-induced myopia may be related to the relationship between the focus in the central and peripheral retina.

Charman and Radhakrishnan ${ }^{40}$ published an extensive review article on the influence of the relative peripheral refraction on ametropia development, which examines both animal and human research. One of the major conclusions of this article was that, although it remains unexplained exactly how relative peripheral hyperopia can control myopia in humans, there is "substantial evidence to suggest that the state of focus in the peripheral retina might have some influence on foveal focus and refraction." $40(\mathrm{p} 334)$

Research in humans has shown that it is not only the fovea that is responsible for the signal causing the eye to accommodate. It has been established that the accommodative response can be triggered by defocus at least 10 degrees out from the fove ${ }^{41}$ and perhaps as far as 30 degrees, ${ }^{42}$ indicating that the peripheral retina is capable of detecting and responding to defocus and therefore of having focus regulation capabilities.

Some clinical trials attempting to control myopia progression in humans using progressive addition spectacle lenses ${ }^{10}$ and contact lenses with either a graduated peripheral power ${ }^{43}$ or Dual Focus bi-zones ${ }^{44}$ have reported a small, statistically significant MDiff $_{\text {ref, }}$ which is encouraging but not yet clinically meaningful. As many years are required to demonstrate a sizeable effect on myopia progression in humans, testing an MPC design in an animal model provides a more timely method to optimize the design, although there are some important points to consider when equating this result in chickens with the human eye, such as differences in accommodative mechanism, ocular shape, scleral structure, and retinal complexity. Some of these differences, however, may be beneficial in producing an enhanced myopia control effect in humans compared with chickens. A more prolate shape of the globe and accommodative lag has been reportedly associated with myopic children and both serve to exaggerate the degree of peripheral defocus. ${ }^{45}$ The Test 2 lens design used in this experiment has been shown to reduce accommodative lag in children when incorporated into a soft contact lens. ${ }^{46}$

Accommodative lag has been suggested as a potential risk factor for myopia; therefore, reducing it could be one of the reasons for achieving positive results for controlling eye growth when specific designs of contact lenses ${ }^{46}$ or spectacles $^{47}$ are used in children. Although it remains uncertain whether the more prolate eye shape and relative peripheral hyperopia in children are causative of myopia or simply associated with it. The study by Sng et al. ${ }^{48}$ on Chinese Singapore children confirmed a link between relative peripheral hyperopia and myopia but could not determine if the association was a causative one. The article by Hoogerheide et al. in $1971^{49}$ was initially understood to show relative peripheral hyperopia as predictive of the development of myopia, but a recent critique of this article has questioned the timing of the data collection thus casting doubt on the predictive assumptions. ${ }^{50}$ A recent study by $S c h m i d{ }^{51}$ investigated retinal steepness changes in children over a 30-month period and concluded that eye shape at the fovea, likely in conjunction with peripheral defocus, was predictive for the increase in ocular axial length. In contrast, a longitudinal study of children from age 8 over 5 years concluded that the degree of relative peripheral hyperopia was not consistently predictive of myopia development. ${ }^{52}$

The large and significant differences in refraction and axial length between the Control and Test lens-wearing eyes, indicate that the two iterations of this MPC lens design have provided a mechanism to control eye growth in chickens. It should be kept in mind that this work investigated the control of defocus-induced myopia, rather than "natural" myopia progression. Future work should further investigate the effect of varying the amount and rate of increase of the progressive plus power in the lens designs, as well as whether or not the designs are capable of decreasing the response to higher amounts of myopic defocus. Measuring off-axis refraction, as well as central, would also be of interest. The 1-week trials of the Test 2 lens design in children have been encouraging. ${ }^{46}$ If this design can be as effective in humans as it has been in this chicken study, the potential for controlling myopia progression is significant.

\section{Acknowledgments}

Supported by a research grant from Visioneering Technologies, Inc., who also supplied the lenses used in the study.

Disclosure: J. Woods, Visioneering Technologies, Inc. (F); S.E. 
Guthrie, Visioneering Technologies, Inc. (F); N. Keir, Visioneering Technologies, Inc. (F); S. Dillehay, Visioneering Technologies, Inc. (F, E); M. Tyson, Visioneering Technologies, Inc. (F, E); R. Griffin, Visioneering Technologies, Inc. (F, E), P; V. Choh, Visioneering Technologies, Inc. (F); D. Fonn, Visioneering Technologies, Inc. (F, C); L. Jones, Visioneering Technologies, Inc. (F); E. Irving, Visioneering Technologies, Inc. (F)

\section{References}

1. World Health Organization. Fact Sheet No. 282. Updated April 2011. Available at: www.who.int/entity/mediacentre/ factsheets/fs282/en. Accessed July 5, 2012.

2. Lai TY, Fan DS, Lai WW, Lam DS. Peripheral and posterior pole retinal lesions in association with high myopia: a crosssectional community-based study in Hong Kong. Eye. 2008;22: 209-213.

3. Xu L, Wang Y, Wang S, Wang Y, Jonas JB. High myopia and glaucoma susceptibility, the Beijing Eye Study. Ophthalmology. 2007; 114:216-220.

4. Lim R, Mitchell P, Cumming RG. Refractive associations with cataract: the Blue Mountains Eye Study. Invest Ophthalmol Vis Sci. 1999; 40:3021-3026.

5. Seet B, Wong TY, Tan DTH, et al. Myopia in Singapore: taking a public health approach. Br J Ophthalmol. 2001;85:521-526.

6. Lin LL, Shih YF, Hsiao CK, Chen CJ. Prevalence of myopia in Taiwanese schoolchildren: 1983-2000. Annals Academy of Medicine. 2004;33:27-33.

7. Vitale S, Sperduto RD, Ferris FL III. Increased prevalence of myopia in the United States between 1971-1972 and 19992004. Arch Ophthalmol. 2009;127:1632-1639.

8. Bates WH. Perfect Sight without Glasses. New York, NY: Central Fixation Publishing Co.; 1920.

9. Goss DA. Effect of bifocal lenses on the rate of childhood myopia progression. Am J Optom Physiol Opt 1986;63:135141.

10. Gwiazda J, Hyman L, Hussein M, et al. A randomized clinical trial of progressive addition lenses versus single vision lenses on the progression of myopia in children. Invest Ophthalmol Vis Sci. 2003;44:1492-1500.

11. Siatkowski RM, Cotter SA, Crockett RS, et al. Two-year multicenter, randomized, double-masked, placebo-controlled, parallel safety and efficacy study of $2 \%$ pirenzepine ophthalmic gel in children with myopia. J AAPOS. 2008;12:332-339.

12. Chung K, Mohidin N, O'Leary DJ. Undercorrection of myopia enhances rather than inhibits myopia progression. Vis Res. 2002; $42: 2555-2559$.

13. Lee T, Cho P. Discontinuation of orthokeratology and myopic progression. Optom Vis Sci. 2010;87:1053-1056.

14. Wiesel TN, Raviola E. Myopia and eye enlargement after neonatal lid fusion in monkeys. Nature. 1977;266:66-68.

15. Sherman SM, Norton TT, Casagrande VA. Myopia in the lidsutured tree shrew (Tupaia glis). Brain Res. 1977;124:154157.

16. Wallman J, Turkel J, Trachtman J. Extreme myopia produced by modest change in early visual experience. Science. 1978; 201:1249-1251.

17. Irving EL, Callender MG, Sivak JG. Inducing myopia, hyperopia and astigmatism in chicks. Optom Vis Sci. 1991;68:364-368.

18. Schaeffel F, Glasser A, Howland HC. Accommodation, refractive error and eye growth in chickens. Vis Res. 1988;28:639657.

19. Wildsoet C, Wallman J. Choroidal and scleral mechanisms of compensation for spectacle lenses in chicks. Vis Res. 1995;35: 1175-1194.
20. Hung L, Crawford MLJ, Smith EL. Spectacle lenses alter the eye growth and the refractive status of young monkeys. Nat Med. 1995; 1:761-765.

21. Norton TT. Animal models of myopia: learning how vision controls the size of the eye. ILAR J. 1999;40:59-77.

22. Irving EL, Sivak JG, Callendar MG. Refractive plasticity of the developing chick eye. Ophthalmic Physiol Opt. 1992;12:448456.

23. Troilo D, Wallman J. The regulation of eye growth and refractive state: an experimental study of emmetropization. Vis Res. 1991;31:1237-1250.

24. Irving EL, Callender MG, Sivak JG. Inducing ametropias in hatchling chicks by defocus-aperture effects and cylindrical lenses. Vis Res. 1995;35:1165-1174.

25. Wallman J, Nickla DL. Chapter 4.1: The relevance of studies in chicks for understanding myopia in humans. In: Beuerman, R, Saw, SM, Tan, DTH, Wong, T, eds. MYOPIA - Animal Models to Clinical Trials. Hackensack, NJ: World Scientific Publishing Co Pte Ltd.; 2010.

26. Smith EL, Hung L-F, Huang J. Relative peripheral hyperopic defocus alters central refractive development in infant monkeys. Vis Res. 2009;49:2386-2392.

27. Tabernero J, Vazquez D, Seidemann A, Uttenweier D, Schaeffel F. Effects of myopic spectacle correction and radial refractive gradient spectacle lenses on peripheral refraction. Vis Res. 2009;49:2176-2186.

28. Glickstein M, Millodot M. Retinoscopy and eye size. Science. 1970;168:605-606.

29. Irving EL, Sivak JG, Curry TA, Callender MG. Chick eye optics: zero to fourteen days. J Comp Physiol A. 1996;179:185-194.

30. Kisilak ML, Hunter JJ, Huang L, et al. In chicks wearing high powered negative lenses, spherical refraction is compensated and oblique astigmatism is induced. J Mod Opt. 2010;55:611623.

31. Stone RA, Pendrak K, Sugimoto R, et al. Local patterns of image degradation differentially affect refraction and eye shape in chick. Curr Eye Res. 2006;31:91-105.

32. Wallman J, Gottleib MD, Rajaram V, Fugate-Wentzek LA. Local retinal regions control local eye growth and myopia. Science. 1987;237:73-77.

33. Liu Y, Wildsoet C. The effect of two-zone concentric bifocal spectacle lenses on refractive error development and eye growth in young chicks. Invest Ophthalmol Vis Sci. 2010;52: 1078-1086.

34. Schippert R, Schaeffel F. Peripheral defocus does not necessarily affect central refractive development. Vis Res. 2006;46:3935-3940.

35. Liu Y, Wildsoet C. The effective add inherent in 2-zone negative lenses inhibits eye growth in myopic young chicks. Invest Ophthalmol Vis Sci. 2012;53:5085-5093.

36. Tepelus TC, Vazquez D, Seidemann A, Uttenweiler D, Schaeffel F. Effects of lenses with different power profiles on eye shape in chickens. Vis Res. 2012;54:12-19.

37. Benavente-Perez A, Nour A, Troilo D. The effect of simultaneous negative and positive defocus on eye growth and development of refractive state in marmosets. Invest Ophthalmol Vis Sci. 2012;53:6479-6487.

38. Schaeffel F, Howland HC, Farkas L. Natural accommodation in the growing chicken. Vis Res. 1986;26:1977-1993.

39. Schaeffel F, Glasser A, Howland HC. Accommodation, refractive error and eye growth in chickens. Vis Res. 1988;28:639657.

40. Charman WN, Radhakrishnan H. Peripheral refraction and the development of refractive error: a review. Ophthalmic Physiol Opt. 2010;30:321-338. 
41. Bullimore MA, Gillmartin B. Retinal eccentricity and the accommodation response. Am J Optom Physiol Opt. 1987;64: 644-645.

42. Gu Y, Legge GE. Accommodation to stimuli in peripheral vision. J Opt Soc Am A. 1987;4:1681-1687.

43. Sankaridurg P, Holden BA, Smith EL, et al. Decrease in rate of myopia progression with a contact lens designed to reduce relative peripheral hyperopia: one-year results. Invest $O p h$ thalmol Vis Sci. 2011;52:9362-9367.

44. Anstice NS, Phillips JR. Effect of dual-focus soft contact lens wear on axial myopia progression in children. Ophthalmology. 2011;118:1152-1161.

45. Mutti DO, Sholtz RI, Friedman NE, Zadnik K. Peripheral refraction and ocular shape in children. Invest Ophthalmol Vis Sci. 2000;41:1022-1030.

46. Miller J, Long B, Dillehay SM. Children's evaluation of a unique myopia progression control lens design. Paper presented at: American Academy of Optometry Conference; October 12-15, 2011; Boston, MA. Abstract 115,896.
47. Gwiazda J, Thorn F, Held R. Accommodation and related risk factors associated with myopia progression and their interaction with treatment in COMET children. Invest Ophthalmol Vis Sci. 2004; 45:2143-2151.

48. Sng CCA, Lin X-Y, Gazzard G, et al. Peripheral refraction in Singapore Chinese children. Invest Ophthalmol Vis Sci. 2011; 52:1181-1189.

49. Hoogerheide J, Rempt F, Hoogenbloom WPH. Acquired myopia in young pilots. Ophthalmologica. 1971;163:209-215.

50. Rosen R, Lundstrom L, Unsbo P, Atchison DA. Have we misinterpreted the study of Hoogerheide et al (1971)? Optom Vis Sci. 2012;89:235-237.

51. Schmid GF. Association between retinal steepness and central myopic shift in children. Optom Vis Sci. 2011;88:684-690.

52. Mutti DO, Sinnott LT, Mitchell GL, et al. Relative peripheral refractive error and the risk of onset and progression of myopia in children. Invest Ophthalmol Vis Sci. 2011;52:199205. 